\title{
Bird Fauna of Jabel El Dayer National Park, Kordofan - Sudan
}

Sanaa E. Ahamed

Received: 19 April, 2021, Accepted: 10 Aug, 2021, Published: 1 Oct, 2021

Department of Zoology, Faculty of Science, University of Kordofan, Sudan

sanaaeltayeb24@gmail.com

\section{Abstract}

Two hundred and seventy-four species of birds belonging to 52 families and 16 orders were recorded at Jabel El Dayer National Park during 2014-2016. The listed birds represented about 29 "\%" of the 938 bird species in the old Sudan. The resident, African migrants and Palearctic migrant's species were 186, 27, 61, respectively. Eighty-three species were recorded breeding during the study period e.g., all species of Weavers and Sparrows, Ciconia abdimii, Columba guinea, Urocolius macrourus, Apus affinis, Coracias abyssinica, Tockus nasutus, Pycnonotus barbatus, Oena capensis, Vanellus tectus and Passer domesticus, Species of Columbidae as; Streptopelia capicola, Streptopelia semitorquata, Streptopelia decipiens were very common near human activities especially Columba guinea where build their nest on roofs of their houses. Species of Ploceidae were usually associated with agricultural fields and springs of water. Order Passeriformes has highly diverse and abundant with 25 families and 161 species were Passerines, and 113 species were Non-passerines. According to IUCN Red List, one species recorded Endangered, five species were Near Threatened, three species were Critically Endangered. The possibility of recording more species was discussed.

Keywords: Bird Fauna, Jabel El Dayer National Park, Kordofan, Identification. 


\section{Introduction}

Bird fauna, the master of air, are the most prominent and widespread species of terrestrial and aquatic vertebrate fauna and occupy most of the global environments (Pomeroy, 1992 and Bibby et al., 1998). The bird fauna of Sudan had varied numbers among different authors. Nickolaus (1987) recorded 938 species of birds. According to Wikipedia (2015), 1013 species of birds are listed in Sudan. Some species of birds may adapt to human habitation and mining environment as they provide easy food and habitat (Martin, 1987 and Smith et al., 2005).

Sudan is located on the main routes used by migratory birds from the three continents Europe, Asia and Africa (Hamed, 1998). Sudan is characterized by diverse climate conditions (Nickolaus, 1987 and UNEP, 2007). A lot of studies in different countries have been carried out on bird fauna in various natural and semi natural ecosystems. Some examples included the countries of India (Guptha et al., 2015), Jordan (Evans et al. 2005), Palestine (Abd Rabou, 2019).

The patchy information available on the birds of the Jabel El Dayer, which is located in North Kordofan State of the Sudan, were due to Cave and Macdonald (1955) and Nickolaus (1987). The study of the area has become a basic necessity after Jabel El Dayer was declared as a National Park. Hence, the aim of the current study is to introduce basic data on the bird fauna of the Park as this will help in any managerial plan of the Park.

\section{Material and Methods}

\section{The Study Area}

The Jabel El Dayer is mostly a massive volcanic rock mountain with a lot of different height peaks (Whiteman, 1971 and IFAD and Police of Protected Wildlife, 2008). It is located on south of North Kordofan State, at El-Rahad Abu Dakana locality (Figure.1). It lies between $12 \circ 28^{\prime \prime}$ and $30^{\circ} 30^{\prime \prime} \mathrm{N}$ and $13 \circ 12^{\prime \prime}$, and $42 \circ 30^{\prime \prime} \mathrm{E}$, covering about 315 "km2". Jabel El Dayer extends about 238 kilometers, 14 kilometers in length, 14 kilometers in width, and about 1413 meters above sea level. It covers poor Savannah climate and environment of high mountain.Such features provided scenery of the geological formations and diversity of plants of unique aesthetic value (IFAD and Police of Protected Wildlife, 2008). Agricultural land stretches flat in all directions around the mountain. Jabel El Dayer 
is easily accessible by motor tracks from the nearby towns. In Jabel El Dayer, three distinct ecological systems: Summits, creeks and water streams and flatland water bodies systems were recognized (Figure. 2,3,4).

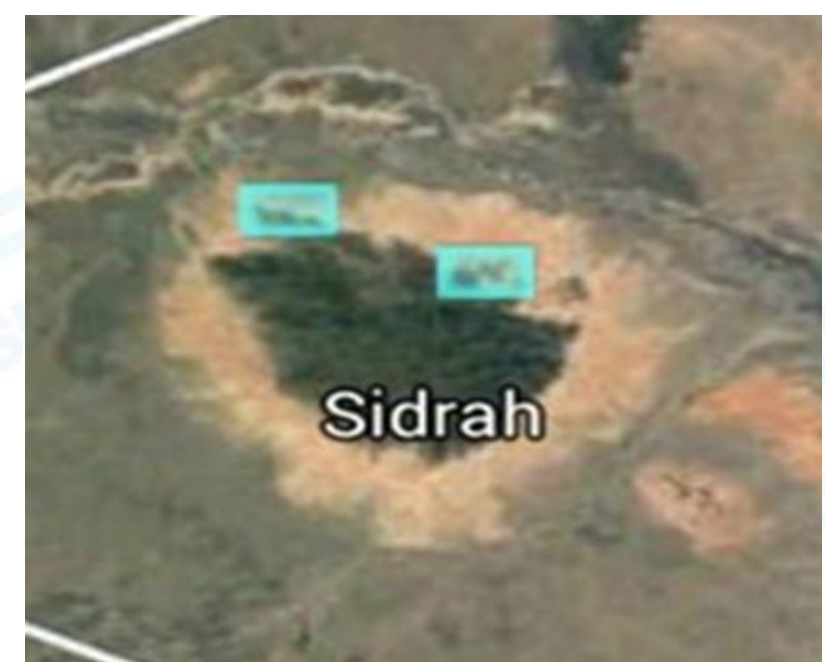

Figure (1): Jabel El Dayer National Park (Http:// www.google.com/earth/).



Figure(2): Jabel El Dayer Study area, note the peaks.

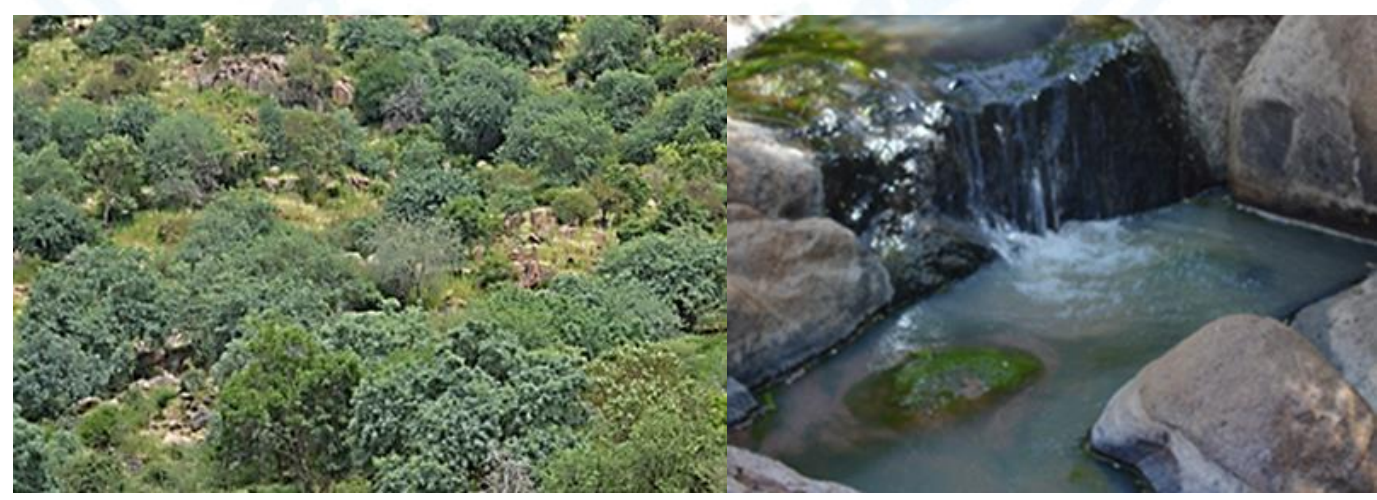

Figure (3): Flatland between peaks.

Figure .4. A water spring (AinTalpas). 


\section{Methods}

The study covered both dry and wet seasons. A total count of birds was conducted twice a day morning and evening. Observations were made in early morning to late afternoon. Many visits were carried out the survey period covered three years from February to December 2014 and from August (2015) to April 2016. Data on breeding activities and nesting habits were collected. The birds were recognized directly and/or with a Pentax $(12 \times 50,5.500)$ binocular. Photographs of birds whenever possible were taken using digital cameras. The Identification of birds was made easy using available guidebooks (Stevenson and Fanshawe, 2002, Scott, 2007 and Sinclair and Ryan, 2010). In spite of all these, some species might have been missed, in view of difficult access, hiding or shyness.

\section{Results and Discussion:}

The bird species recorded in both seasons in Jabel El Dayer National Park were given in Table (1). Their status information $(\mathrm{B}=$ Breeding record confirmed; $\mathrm{M}=$ Migrant including on passage through Sudan; $\mathrm{P}=$ Breeds in Palearctic; $\mathrm{R}=$ Resident and $\mathrm{W}=$ Winters non-breeding season) followed Dowsett et al. (2014). The study recorded 274 species belonging to 52 families and 16 orders. During the first season 263 species and 202 species during the second season were recorded at the Park. Some species were recorded breeding during the study period e.g. Ciconia abdimii, Columba guinea, Apus affinis, Coracias abyssinica (Figure 4). The Ptilostomus afer, Corvus capensis, Passer domesticus, Euodice cantans (Figure 5) were very common near human activities. Members of families Ploceidae and Columbidae (Figure 6) were usually associated with agricultural fields and springs of water while Gyps rueppellii, Circus pygargus and Ptilopachus petrosus were seen in clefts at top of mountain only (Figure 7). 


\section{ISRAA UNIVERSITY JOURNAL OF APPLIED SCIENCE}
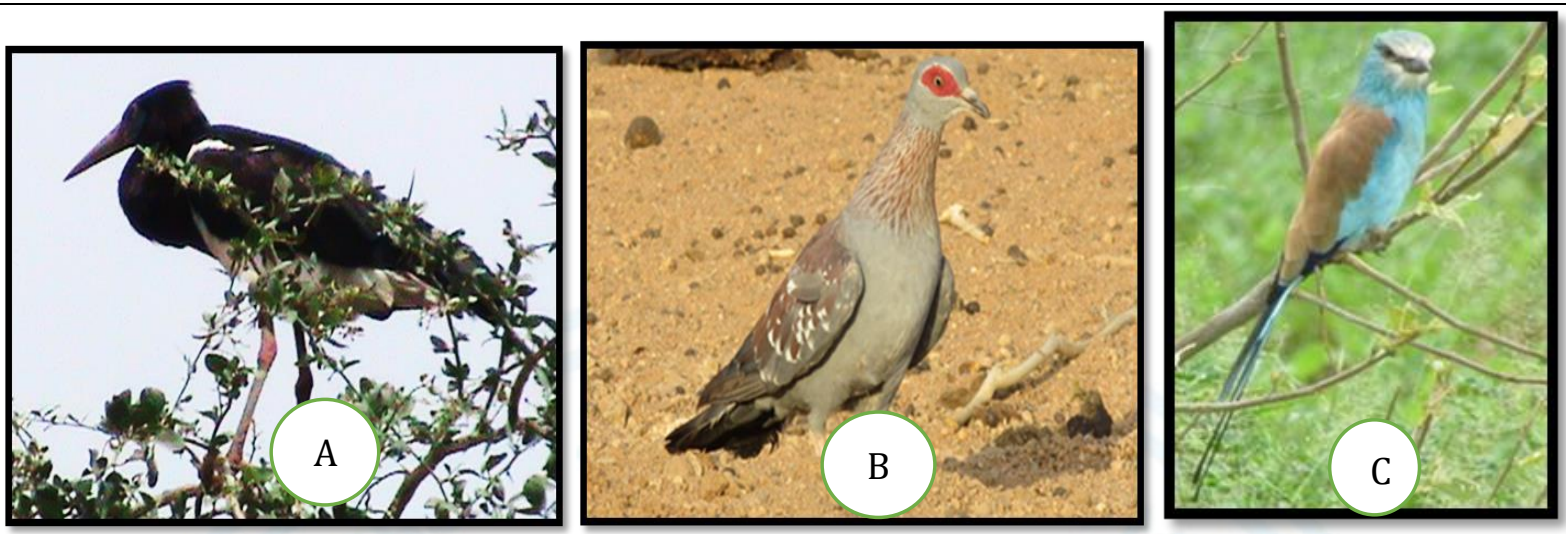

Figure (4): A. Abdim's Stork (Ciconia abdimii), B. Speckled Pigeon (Columba guinea), C. Abyssinian Roller (Coracias abyssinica), D. Red-billed Horn bill (Tockus erythrorhynchus), E. Vieillot's Barbet (Lybius vieilloti), F. Northern Red Bishop (Euplectes franciscanus).
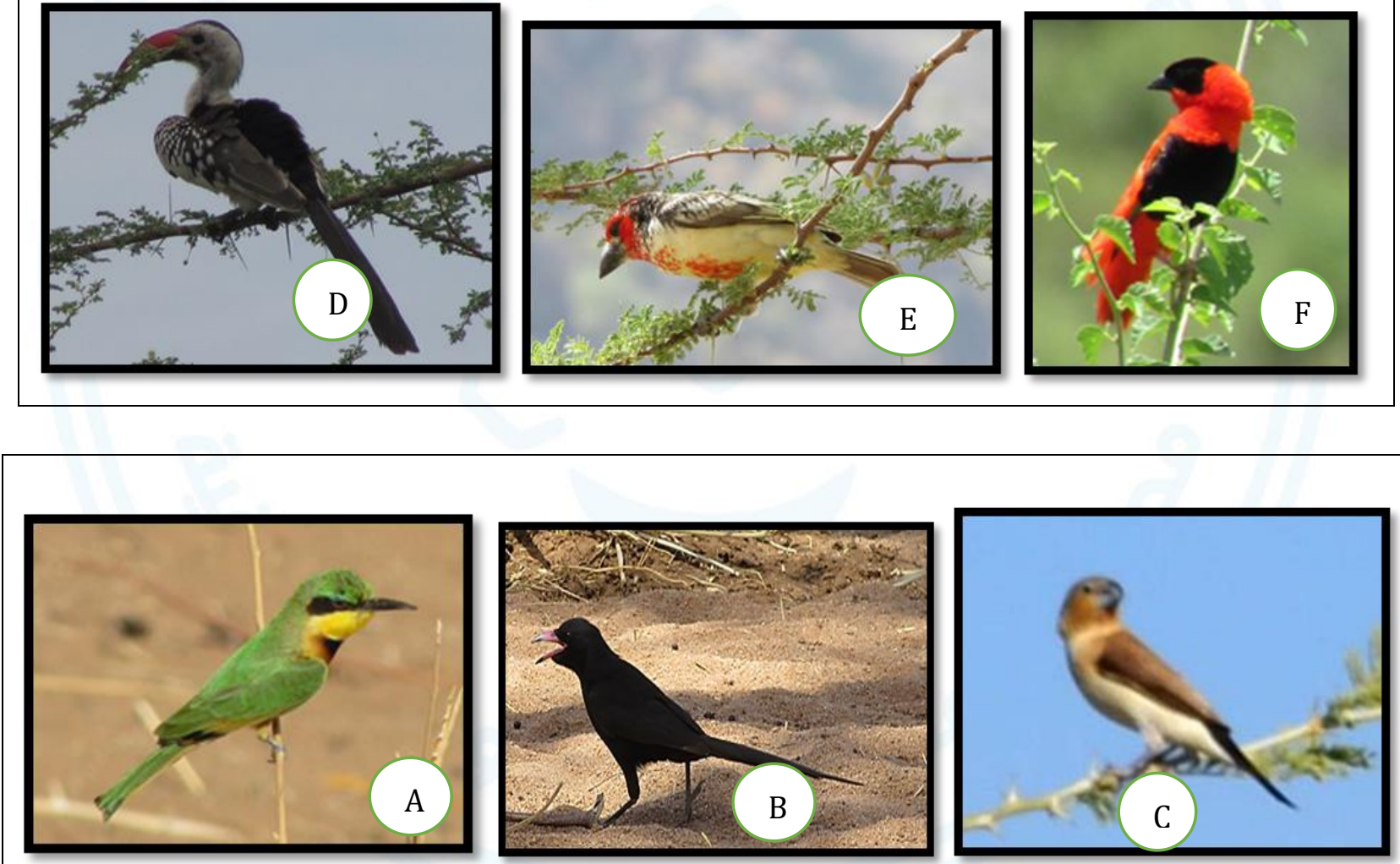

Figure (5): A. Little Bee-eater (Merops pusillus), B. Pipiac (Ptilostomus afer), C. African Silver bill (Euodice cantans).

Table (1): The Status (ST) of Birds of Jabel El Dayer National Park.

\begin{tabular}{|l|l|l|l|c|}
\hline Common name & Scientific name & 2014 & $2015 / 2016$ & ST \\
\hline Order Ciconiiformes: 1- Family: Ardeidae & Bubulcus ibis & + & + & M+B+P \\
\hline Cattle Egret*
\end{tabular}




\begin{tabular}{|c|c|c|c|c|}
\hline Little Egret & Egrettagarzetta & + & - & $\mathrm{R}+\mathrm{B}+\mathrm{P}$ \\
\hline Grey Heron & Ardea cinerea & + & - & $\mathrm{R}+\mathrm{B}+\mathrm{P}$ \\
\hline \multicolumn{5}{|c|}{ Order Ciconiiformes: 2- Family: Ciconiidae } \\
\hline Abdim's Stork* & Ciconia abdimii & + & + & $\mathrm{M}+\mathrm{B}$ \\
\hline White Stork & Ciconia ciconia & + & - & $\mathrm{P}+\mathrm{W}$ \\
\hline \multicolumn{5}{|c|}{ OrderFalconiformes: 1- Family: Accipiteridae } \\
\hline Black Kite & Milvus migrans & + & + & $\mathrm{M}+\mathrm{B}+\mathrm{P}+\mathrm{W}$ \\
\hline African White-backed Vulture* & Gyps africanus & + & + & $\mathrm{R}+\mathrm{B}$ \\
\hline Ruppell,s Griffon Vulture* & Gyps rueppellii & - & + & $\mathrm{R}+\mathrm{B}$ \\
\hline Hooded Vulture & Necrosyrtes monachus & + & - & $\mathrm{R}+\mathrm{B}$ \\
\hline Short-toed Snake-Eagle & Circaetus gallicus & + & - & $\mathrm{P}+\mathrm{W}+\mathrm{M}+\mathrm{B}$ \\
\hline Brown Snake-Eagle & Circaetus cinereus & + & - & $\mathrm{R}+\mathrm{B}$ \\
\hline African Marsh Harrier & Circus ranivorus & + & + & $\mathrm{R}+\mathrm{M}$ \\
\hline Eurasian Marsh Harrier & Circus aeruginosus & + & - & $\mathrm{P}+\mathrm{W}$ \\
\hline Montagu,s Harrier & Circus pygargus & + & + & $\mathrm{P}+\mathrm{W}$ \\
\hline Pallid Harrier & Circus macrourus & + & + & $\mathrm{P}+\mathrm{W}$ \\
\hline Dark Chanting-Goshawk & Melierax metabates & + & + & $\mathrm{R}+\mathrm{B}$ \\
\hline Gabar Goshawk* & Melierax gabar & + & + & $\mathrm{R}+\mathrm{B}$ \\
\hline Shikra & Accipiter badius & + & + & $\mathrm{R}+\mathrm{B}$ \\
\hline Little Sparrow Hawk & Accipiter minullus & + & + & $\mathrm{R}$ \\
\hline Great Sparrow Hawk & Accipiter melanoleucus & + & - & $\mathrm{R}$ \\
\hline Bat Hawk & Macheiramphus alcinus & + & - & $\mathrm{R}$ \\
\hline African Harrier Hawk & Polyboroides typus & + & - & $\mathrm{R}+\mathrm{B}$ \\
\hline Long-tailed Hawk & Urotriorchis macrourus & + & - & $\mathrm{R}+\mathrm{M}$ \\
\hline lizard Buzzard & Kaupifalcomonogrammicus & + & + & $\mathrm{R}+\mathrm{B}$ \\
\hline Grasshopper Buzzard & Butastur rufipennis & + & - & $\mathrm{M}+\mathrm{B}$ \\
\hline Mountain Buzzard & oreophilus & + & + & $\mathrm{R}$ \\
\hline Common Buzzard & Buteo & + & + & $\mathrm{P}+\mathrm{M}$ \\
\hline Long-legged Buzzard & rufinus & + & + & $\mathrm{P}+\mathrm{W}+\mathrm{R}$ \\
\hline Tawny Eagle & Aquila rapax & + & + & $\mathrm{M}+\mathrm{B}$ \\
\hline Steppe Eagle & Aquila nipalensis & + & - & $\mathrm{P}$ \\
\hline \multicolumn{5}{|c|}{ OrderFalconiformes: 2- Family: Falconidae } \\
\hline Common Kestrel & Falco tinnunculus & + & + & $\mathrm{R}+\mathrm{B}+\mathrm{P}+\mathrm{W}$ \\
\hline Lesser Kestrel & Falco naumanni & + & + & $\mathrm{P}$ \\
\hline Fox Kestrel & Falco alopex & + & + & $\mathrm{R}+\mathrm{B}$ \\
\hline Grey Kestrel & Falco ardosiaceus & - & + & $\mathrm{R}+\mathrm{B}$ \\
\hline African Hobby & Falco cuvieri & + & + & $\mathrm{R}$ \\
\hline Sooty Falcon & Falcoconcolor & + & + & $\mathrm{P}$ \\
\hline Lanner Falcon & Falco biarmicus & + & + & $\mathrm{M}+\mathrm{B}+\mathrm{P}$ \\
\hline Red-footed Falcon & Falco vespertinus & + & - & $\mathrm{P}$ \\
\hline Peregrine Falcon & Falco peregrinus & + & + & $\mathrm{R}+\mathrm{B}+\mathrm{P}$ \\
\hline Pygmy Falcon & Polihierax semitorquatus & + & - & $\mathrm{R}+\mathrm{M}$ \\
\hline \multicolumn{5}{|c|}{ Order Galliformes: 1- Family: Numididae } \\
\hline Helmeted Guinea fowl & Numida meleagris & + & + & $\mathrm{R}+\mathrm{B}$ \\
\hline \multicolumn{5}{|c|}{ Order Galliformes: 2- Family: Phasianidae } \\
\hline Clapperton,s Francolin & Francolinus clappertoni & + & - & $\mathrm{R}+\mathrm{B}$ \\
\hline Common Quail & Coturnix coturnix & + & + & $\mathrm{P}+\mathrm{W}$ \\
\hline Stone Partridge & Ptilopachus petrosus & + & + & $\mathrm{R}+\mathrm{B}$ \\
\hline \multicolumn{5}{|c|}{ OrderGruiformes: Family: Otididae } \\
\hline White-billed Bustard & Eupodotis senegalensis & + & - & $\mathrm{R}+\mathrm{B}$ \\
\hline Buff-crested Bustard & Eupodotis gindiana & + & - & $\mathrm{R}+\mathrm{B}$ \\
\hline \multicolumn{5}{|c|}{ OrderCharadiiformes: 1- Family: Burhinidae } \\
\hline Spotted Thick-knee & Burhincus & + & + & $\mathrm{R}+\mathrm{B}$ \\
\hline
\end{tabular}




\begin{tabular}{|c|c|c|c|c|}
\hline Senegal Thick-knee & Burhinus senegalensis & - & + & $\mathrm{R}+\mathrm{B}$ \\
\hline \multicolumn{5}{|c|}{ Order Charadiiformes: 2- Family: Charadriidae } \\
\hline Black-headed Plover* & Vanellus & + & + & $\mathrm{R}+\mathrm{B}$ \\
\hline Spur-winged Plover & Vanellus spinosus & + & - & $\mathrm{R}+\mathrm{B}$ \\
\hline \multicolumn{5}{|c|}{ Order Pterocliformes : Family: Pteroclidae } \\
\hline Chestnut-bellied Sand Grouse & Pterocles exustus & + & + & $\mathrm{R}+\mathrm{B}$ \\
\hline \multicolumn{5}{|c|}{ Order Columbiformes: Family: Columbidae } \\
\hline Bruce's Green-pigeon & Treron waalia & + & + & $\mathrm{R}+\mathrm{B}$ \\
\hline Speckled Pigeon* & Columba guinea & + & + & $\mathrm{R}+\mathrm{B}$ \\
\hline Black-billed Wood Dove* & Turtur abyssinicus & + & + & $\mathrm{R}+\mathrm{B}$ \\
\hline Namaqua Dove* & Oena capensis & + & + & $\mathrm{M}+\mathrm{B}$ \\
\hline Laughing Dove* & Streptopelia senegalensis & + & + & $\mathrm{R}+\mathrm{B}$ \\
\hline Ring-necked Dove* & Streptopelia capicola & + & + & $\mathrm{R}$ \\
\hline Red-eyed Dove* & Streptopelia semitorquata & + & + & $\mathrm{R}+\mathrm{B}$ \\
\hline Mourning Dove* & Streptopelia decipiens & + & + & $\mathrm{R}+\mathrm{B}$ \\
\hline Pink-headed Dove* & Streptopelia roseogrisea & + & + & $\mathrm{R}+\mathrm{B}$ \\
\hline Vinaceous Dove & Streptopelia vinacea & + & - & $\mathrm{R}+\mathrm{B}$ \\
\hline \multicolumn{5}{|c|}{ Order Psittaciformes: Family: Psittacidae } \\
\hline Brown Parrot & Poicephalus meyeri & + & + & $\mathrm{R}+\mathrm{B}$ \\
\hline Rose-ringed Parakeet & Psittacula krameri & + & + & $\mathrm{R}+\mathrm{B}$ \\
\hline \multicolumn{5}{|c|}{ Order Cuculiformes: Family: Cuculidae } \\
\hline Great Spotted Cuckoo & Clamator glandarius & + & - & $\mathrm{M}+\mathrm{B}$ \\
\hline African Cuckoo & Cuculus gularis & + & + & $\mathrm{M}+\mathrm{B}$ \\
\hline Eurasian Cuckoo & Cuculus canorus & - & + & $\mathrm{P}$ \\
\hline Diederik cuckoo & Chrysococcyx caprius & + & + & $\mathrm{M}+\mathrm{B}$ \\
\hline Senegal Coucal & Centropus senegalensis & + & - & $\mathrm{R}+\mathrm{B}$ \\
\hline White-browed Coucal & Centropus superciliolsus & + & + & $\mathrm{R}+\mathrm{B}$ \\
\hline \multicolumn{5}{|c|}{ OrderStrigiformes: 1- Family: Tytonidae } \\
\hline Spotted Eagle Owl & africanus & + & - & $\mathrm{R}+\mathrm{B}$ \\
\hline Verreaux,s Eagle Owl & Bubo lacteus & + & - & $\mathrm{R}+\mathrm{B}$ \\
\hline Barn Owl & Tyto alba & + & + & $\mathrm{R}+\mathrm{B}$ \\
\hline Pearl-spotted Owlet & Glaucidium perlatum & + & - & $\mathrm{R}+\mathrm{B}$ \\
\hline \multicolumn{5}{|c|}{ OrderStrigiformes: 2- Family: Strigidae } \\
\hline White-faced Scops-Owl & leucotis & + & + & $\mathrm{R}+\mathrm{B}$ \\
\hline \multicolumn{5}{|c|}{ Order Caprimulgiformes: Family: Caprimulgidae } \\
\hline Long-tailed Nightjar & Caprimulgus climacurus & + & + & $\mathrm{M}+\mathrm{B}$ \\
\hline Nubian Nightjar & Caprimulgus nubicus & + & - & $\mathrm{R}$ \\
\hline Golden Nightjar & Caprimulgus & + & - & $\mathrm{R}+\mathrm{B}$ \\
\hline Standard-winged Nightjar & Macrodipteryx longipennis & + & - & $\mathrm{M}+\mathrm{B}$ \\
\hline \multicolumn{5}{|c|}{ Order Apodiformes: Family: Apodidae } \\
\hline Little Swift* & Apus affinis & + & + & $\mathrm{R}+\mathrm{B}$ \\
\hline Mottled Swift* & Tachymarptis aequatorialis & + & + & $\mathrm{R}+\mathrm{B}$ \\
\hline Eurasian Swift & Apus apus & + & + & $\mathrm{P}$ \\
\hline Horus Swift* & Apus horus & + & + & $\mathrm{R}+\mathrm{B}$ \\
\hline Scarce Swift & Schoutedenapus myoptilus & + & + & $\mathrm{R}+\mathrm{B}$ \\
\hline African Palm Swift* & Cypsiurus & + & + & $\mathrm{R}+\mathrm{B}$ \\
\hline \multicolumn{5}{|c|}{ Order Colliformes: Family: Collidae } \\
\hline Speckled Mouse bird* & Colius striatus & + & + & $\mathrm{R}+\mathrm{B}$ \\
\hline Blue -naped Mouse bird* & Urocolius macrourus & + & + & $\mathrm{R}+\mathrm{B}$ \\
\hline \multicolumn{5}{|c|}{ Order Coreciiformes: 1- Family: Alcedinidae } \\
\hline Grey-headed Kingfisher & Halcyonleucocephala & + & + & $\mathrm{M}+\mathrm{B}$ \\
\hline Woodland Kingfisher & Halcyon senegalensis & + & + & $\mathrm{M}+\mathrm{B}$ \\
\hline Striped kingfisher & Halcyon chelicuti & + & - & $\mathrm{R}+\mathrm{B}$ \\
\hline
\end{tabular}


Order Coreciiformes: 2- Family: Meropidae

\begin{tabular}{|c|c|c|c|c|}
\hline Little Bee-eater* & Meropspusillus & + & + & $\mathrm{R}+\mathrm{B}$ \\
\hline Little Green Bee-eater* & Merops orientalis & + & + & $\mathrm{R}+\mathrm{B}$ \\
\hline White-throated Bee-eater & Merops albicollis & + & + & $\mathrm{M}+\mathrm{B}$ \\
\hline European Bee-eater & Merops apiaster & + & - & $\mathrm{P}$ \\
\hline \multicolumn{5}{|c|}{ Order Coreciiformes: 3- Family: Coraciidae } \\
\hline Abyssinian Roller* & Coracias abyssinica & + & + & $\mathrm{M}+\mathrm{B}$ \\
\hline European Roller & Coracias garrulous & + & + & $\mathrm{P}$ \\
\hline Rufous-crowned Roller* & Coracias naevia & + & + & $\mathrm{M}+\mathrm{B}$ \\
\hline \multicolumn{5}{|c|}{ Order Coreciiformes: 4- Family: Bucerotidae } \\
\hline Grey Horn bill* & Tockus nasutus & + & + & $\mathrm{R}+\mathrm{B}$ \\
\hline Red-billed Horn bill* & Tockus erythrorhynchus & + & + & $\mathrm{R}+\mathrm{B}$ \\
\hline \multicolumn{5}{|c|}{ Order Coreciiformes: 5- Family: Upupidae } \\
\hline African Hoopoe & Africana & + & - & $\mathrm{R}+\mathrm{B}$ \\
\hline Eurasian Hoopoe & Upupa epops & + & + & $\mathrm{R}+\mathrm{B}+\mathrm{P}+\mathrm{W}$ \\
\hline \multicolumn{5}{|c|}{ Order Coreciiformes: 6- Family: Phoeniculidae } \\
\hline Green Wood-hoopoe* & Phoeniculus purpureus & + & + & $\mathrm{R}+\mathrm{B}$ \\
\hline Black Wood-hoopoe* & Phoeniculus somaliensis & + & + & $\mathrm{R}$ \\
\hline Abyssinian Scimitarbill & Rhiopomastus minor & + & - & $\mathrm{R}+\mathrm{B}$ \\
\hline \multicolumn{5}{|c|}{ Order Piciformes: 1- Family: Capitonidae } \\
\hline Vieillot's Barbet* & Lybius vieilloti & + & + & $\mathrm{R}+\mathrm{B}$ \\
\hline black-breasted Barbet & Lybius rolleti & + & - & $\mathrm{R}+\mathrm{B}$ \\
\hline Yellow- breasted Barbet* & Trachyphonus margaritatus & + & + & $\mathrm{R}+\mathrm{B}$ \\
\hline \multicolumn{5}{|c|}{ Order Piciformes: 2- Family: Indictoridae } \\
\hline Black-throated Honey guide & Indicator indicator & + & - & $\mathrm{R}$ \\
\hline Lesser Honey guide & Indicator minor & + & + & $\mathrm{R}$ \\
\hline \multicolumn{5}{|c|}{ Order Piciformes: 3- Family: Picidae } \\
\hline Brown-backed Wood Pecker & Picoides obsoletus & + & - & $\mathrm{R}+\mathrm{B}$ \\
\hline Nubian Wood Pecker & Campethera nubica & + & + & $\mathrm{R}+\mathrm{B}$ \\
\hline Bearded wood Pecker & Dendropicos namaquus & + & + & $\mathrm{R}+\mathrm{B}$ \\
\hline Grey Wood Pecker & Dendropicos goertae & + & + & $\mathrm{R}+\mathrm{B}$ \\
\hline Little Grey Wood Pecker & Dendropicos elachus & + & _- & $\mathrm{R}+\mathrm{B}$ \\
\hline \multicolumn{5}{|c|}{ Order Passeriformes: 1- Family: Alaudidae } \\
\hline Red-winged Lark & Mirafra hypermetra & + & - & $\mathrm{R}$ \\
\hline Flappet Lark & Mirafra rufocinnamomea & + & - & $\mathrm{R}+\mathrm{B}$ \\
\hline Singing Bush Lark & Mirafra cantillans & + & - & $\mathrm{R}+\mathrm{B}$ \\
\hline Sun Lark & Galerida modesta & + & - & $\mathrm{R}+\mathrm{B}$ \\
\hline Short-toed Lark & Calandrella brachydactyla & + & + & $\mathrm{P}+\mathrm{W}$ \\
\hline Chestnut-backed Sparrow Lark & Eremopterix leucotis & + & + & $\mathrm{R}+\mathrm{B}$ \\
\hline Chestnut-headed Sparrow Lark & Eremopterix signata & + & - & $\mathrm{R}+\mathrm{B}$ \\
\hline White- fronted Sparrow Lark & Eremopterix nigriceps & + & + & $\mathrm{R}+\mathrm{B}$ \\
\hline \multicolumn{5}{|c|}{ Order Passeriformes: 2- Family: Hirundinidae } \\
\hline Common House Martin & Delichon urbica & + & - & $\mathrm{P}+\mathrm{W}$ \\
\hline African Sand Martin & Riparia paludicola & + & - & $\mathrm{P}+\mathrm{W}$ \\
\hline African Rock Martin & Hirundo fuligula & + & - & $\mathrm{R}+\mathrm{B}+\mathrm{P}+\mathrm{W}$ \\
\hline Eurasian Swallow* & Hirundo rustica & + & + & $\mathrm{P}$ \\
\hline Wire-tailed Swallow* & Hirundo smithii & + & + & $\mathrm{R}+\mathrm{B}$ \\
\hline Ethiopian Swallow* & Hirundo aethiopica & + & + & $\mathrm{R}+\mathrm{B}$ \\
\hline Mosque Swallow & Cecropis senegalensis & + & - & $\mathrm{R}+\mathrm{B}$ \\
\hline \multicolumn{5}{|c|}{ Order Passeriformes: 3- Family: Motacillidae } \\
\hline White wagtail & Motacilla alba & + & + & $\mathrm{P}+\mathrm{W}$ \\
\hline Grey Wagtail & Motacilla cinerea & + & + & $\mathrm{P}+\mathrm{W}$ \\
\hline African Pied Wagtail & Motacilla aguimp & + & - & $\mathrm{R}$ \\
\hline Yellow Wagtail & Motacilla flava & + & + & $\mathrm{P}+\mathrm{W}$ \\
\hline
\end{tabular}




\begin{tabular}{|c|c|c|c|c|}
\hline Tree Pipit & Anthus trivialis & + & - & $\mathrm{P}+\mathrm{W}$ \\
\hline Long-billed Pipit & Anthus similis & + & - & $\mathrm{R}+\mathrm{B}$ \\
\hline \multicolumn{5}{|c|}{ Order Passeriformes: 4- Family: Pycnonotidae } \\
\hline Common Bulbul & Pycnonotus barbatus & + & + & $\mathrm{R}+\mathrm{B}$ \\
\hline Yellow-vented Bulbul & Pycnonotus tricolor & + & + & $\mathrm{R}+\mathrm{B}$ \\
\hline \multicolumn{5}{|c|}{ OrderPasseriformes: 5- Family: Turdidae } \\
\hline Common Rock-thrush & Monticola saxatilis & + & + & $\mathrm{P}+\mathrm{W}$ \\
\hline Blue Rock-Thrush & Monticola solitaries & + & + & $\mathrm{P}+\mathrm{W}$ \\
\hline Northern Anteater Chat & $\begin{array}{l}\text { Myrmecocichla aethiops } \\
\text { sudanensi }\end{array}$ & + & + & $\mathrm{R}+\mathrm{B}$ \\
\hline Ant Chat & Myrmecocichla aethiops & + & + & $\mathrm{R}+\mathrm{B}$ \\
\hline White-crowned Cliff Chat & Thamnolaea cinnamomeiventris & + & + & $\mathrm{R}+\mathrm{B}$ \\
\hline Common Stone Chat & Saxicola torquata & + & + & $\mathrm{R}+\mathrm{B}+\mathrm{P}+\mathrm{W}$ \\
\hline Nightingale & Lusciniamegarhynchos & + & + & $\mathrm{P}+\mathrm{W}$ \\
\hline Common Red start & Phoenicurus phoenicurus & + & + & $\mathrm{P}+\mathrm{W}$ \\
\hline Brown-tailed Rock Chat & Cercomela scotocerca & + & + & $\mathrm{R}+\mathrm{B}$ \\
\hline Red -tailed chat & Cercomela familiaris & + & + & $\mathrm{R}$ \\
\hline Black-tailed Rock Chat & Cercomela melanura & + & + & $\mathrm{R}+\mathrm{B}$ \\
\hline Rufous Bush Chat & Cercotrichas galactotes & + & + & $\mathrm{R}+\mathrm{B}+\mathrm{P}+\mathrm{W}$ \\
\hline Black Bush Robin* & Cercotrichas podobe & + & + & $\mathrm{R}+\mathrm{B}$ \\
\hline White-browed Robin-Chat & Cossypha heuglini & + & + & $\mathrm{R}+\mathrm{B}$ \\
\hline Snowy-headed Robin-Chat & Cossypha niveicapilla & + & + & $\mathrm{R}+\mathrm{B}$ \\
\hline Northern Wheatear & Oenanthe oenanthe & + & + & $\mathrm{P}+\mathrm{W}$ \\
\hline Isabelline Wheatear & Oenanthe isabellina & + & + & $\mathrm{P}+\mathrm{W}$ \\
\hline Pied Wheatear & Oenanthe pleschanka & + & + & $\mathrm{P}+\mathrm{W}$ \\
\hline Black - eared Wheatear & Oenanthe hispanica & + & + & $\mathrm{P}+\mathrm{W}$ \\
\hline Desert Wheatear & Oenanthe deserti & + & + & $\mathrm{P}+\mathrm{W}$ \\
\hline Red- breasted Wheatear & Oenanthe heuglini & + & - & $\mathrm{R}+\mathrm{B}$ \\
\hline \multicolumn{5}{|c|}{ Order Passeriformes: 6- Family: Sylviidae } \\
\hline African Reed Warbler & Acrocephalus baeticatus & + & + & $\mathrm{M}+\mathrm{B}$ \\
\hline Sedge Warbler & Acrocephalus schoenobaenus & + & - & $\mathrm{P}+\mathrm{W}$ \\
\hline Reed Warbler & Acrocephalus scirpaceus & + & + & $\mathrm{P}+\mathrm{W}+\mathrm{M}+\mathrm{B}$ \\
\hline Willow Warbler & Phylloscopus trochilus & + & - & $\mathrm{P}$ \\
\hline Wood Warbler & Phylloscopus sibilatrix & + & + & $\mathrm{P}+\mathrm{W}$ \\
\hline Acacia Warbler & Phyllolais pulchellus & + & + & \\
\hline Upcher,s Warbler & Hippolais languida & + & - & $\mathrm{P}+\mathrm{V}$ \\
\hline Olivaceous Warbler & Hippolais pallida & + & + & $\mathrm{R}+\mathrm{B}+\mathrm{P}+\mathrm{W}$ \\
\hline Cricket Warbler & Spiloptila clamans & - & + & $\mathrm{R}+\mathrm{B}$ \\
\hline Rufous Warbler & Erythropygia rufus & + & - & $\mathrm{R}$ \\
\hline Garden Warbler & Sylviaborin & + & - & $\mathrm{P}$ \\
\hline Barred Warbler & Sylvianisoria & + & - & $\mathrm{P}$ \\
\hline Ruppell,s Warbler & Sylvia rueppelli & + & - & $\mathrm{P}+\mathrm{W}$ \\
\hline Black Cap & Sylvia atricapilla & + & - & $\mathrm{P}+\mathrm{W}$ \\
\hline Common white Throat & Sylvia communis & + & - & $\mathrm{P}+\mathrm{W}$ \\
\hline Crombec* & Sylvietta brachyura & + & + & $\mathrm{R}+\mathrm{B}$ \\
\hline Green-backed Eremomela & Eremomela pusilla & + & + & $\mathrm{R}+\mathrm{B}$ \\
\hline Yellow-billed Eremomela* & Eremomela icteropygialis & + & + & $\mathrm{R}+\mathrm{B}$ \\
\hline Brown -backed Eremomela & Eremomela griseoflava & + & - & $\mathrm{R}+\mathrm{B}$ \\
\hline Order Passeriformes: 7- Family: & Cisticolidae & & & \\
\hline Red-fronted Apalis & Apalis rufifrons & + & - & $\mathrm{R}$ \\
\hline Grey-backed Camaroptera* & Camaroptera brachyura & + & + & $\mathrm{R}+\mathrm{B}$ \\
\hline Desert Cisticola & Cisticola & + & + & $\mathrm{R}+\mathrm{B}$ \\
\hline Zitting Cisticola & Cisticola juncidis & + & + & $\mathrm{R}+\mathrm{B}$ \\
\hline Red-pate Cisticola & Cisticola ruficeps & + & + & $\mathrm{R}+\mathrm{B}$ \\
\hline
\end{tabular}




\begin{tabular}{|c|c|c|c|c|}
\hline Foxy Cisticola & Cisticola troglodytes & + & + & $\mathrm{R}+\mathrm{B}$ \\
\hline Croaking Cisticola & Cisticola natalensis & + & + & $\mathrm{R}$ \\
\hline Tawny-flanked Prinia* & Prinia subflava & - & + & $\mathrm{R}+\mathrm{B}$ \\
\hline \multicolumn{5}{|c|}{ Order Passeriformes: 8- Family: Muscicapidae } \\
\hline Pale Flycatcher & Bradornis pallidus & + & + & $\mathrm{R}+\mathrm{B}$ \\
\hline Spotted Flycatcher & Muscicapa striata & + & + & $\mathrm{P}$ \\
\hline Order Passeriformes: 9- Family: & Monarchidae & & & \\
\hline African Paradise-Flycatcher* & Terpsiphone viridis & + & + & $\mathrm{M}+\mathrm{B}$ \\
\hline \multicolumn{5}{|c|}{ OrderPasseriformes: 10- Family: Platysteiridae } \\
\hline Black-headed Batis* & Batis minor & + & + & $\mathrm{R}+\mathrm{B}$ \\
\hline Grey -headed Batis* & Batis orientalis & + & + & $\mathrm{R}+\mathrm{B}$ \\
\hline \multicolumn{5}{|c|}{ OrderPasseriformes: 11- Family: Paridae } \\
\hline Black Tit & Parus leucomelas & + & + & $\mathrm{R}+\mathrm{B}$ \\
\hline Yellow White-eye & Zosterops senegalensis & + & - & $\mathrm{R}+\mathrm{B}$ \\
\hline \multicolumn{5}{|c|}{ OrderPasseriformes: 12- Family: Remizidae } \\
\hline Sennar Penduline Tit* & Remiz punctifrons & + & + & $\mathrm{R}+\mathrm{B}$ \\
\hline Cappoc Penduline Tit* & Remiz punctiforms & - & + & $\mathrm{R}+\mathrm{B}$ \\
\hline Mouse-coloured Penduline Tit & Remiz musculus & - & + & $\mathrm{R}$ \\
\hline Yellow Penduline Tit & Remiz parvulus & - & + & $\mathrm{R}$ \\
\hline \multicolumn{5}{|c|}{ Order Passeriformes: 13- Family: Nectoriniidae } \\
\hline Scarlet-chested Sunbird* & Nectarinia senegalensis & + & + & $\mathrm{R}+\mathrm{B}$ \\
\hline Beautiful Sun bird* & Nectarinia pulchellus & + & + & $\mathrm{R}+\mathrm{B}$ \\
\hline Copper Sunbird & Nectarinia cupreus & + & + & $\mathrm{R}+\mathrm{B}$ \\
\hline Variable Sunbird & Nectarinia venustus & + & + & $\mathrm{R}+\mathrm{B}$ \\
\hline Pygmy Sun bird* & Anthreptes platura & + & + & $\mathrm{R}+\mathrm{B}$ \\
\hline Eastern Pygmy Sun bird* & Anthreptes metallica & + & + & $\mathrm{R}+\mathrm{B}$ \\
\hline \multicolumn{5}{|c|}{ Order Passeriformes: 14- Family: Laniidae } \\
\hline Grey-backed Fiscal & Laniusexcubitoroides & + & + & $\mathrm{R}+\mathrm{B}$ \\
\hline Great Grey Shrike & Lanius excubitor & + & - & $\mathrm{P}$ \\
\hline Lesser Grey Shrike & Lanius minor & + & + & $\mathrm{P}$ \\
\hline Wood Chat Shrike & Lanius senator & + & + & $\mathrm{P}+\mathrm{W}$ \\
\hline Masked Shrike & Lanius nubicus & + & + & $\mathrm{P}+\mathrm{W}$ \\
\hline Red-tailed Shrike & Lanius isabellinus & + & + & $\mathrm{P}+\mathrm{W}$ \\
\hline Red-backed Shrike & Lanius collurio & + & + & $\mathrm{P}$ \\
\hline Yellow-billed Shrike & Corvinella corvine & + & + & $\mathrm{R}+\mathrm{B}$ \\
\hline \multicolumn{5}{|c|}{ Order Passeriformes: 15- Family: Malacontidae } \\
\hline Black-headed Gonolek & Laniarius erythrogaster & + & - & $\mathrm{R}+\mathrm{B}$ \\
\hline Northern Brubru & Nilaus afer & + & + & $\mathrm{R}+\mathrm{B}$ \\
\hline Northern Puffback & Dryoscopus gambensis & + & - & $\mathrm{R}+\mathrm{B}$ \\
\hline Black-headed Tchagra & Tchagra senegala & + & + & $\mathrm{R}+\mathrm{B}$ \\
\hline Rosy-patched Shrike & Rhodophoneus cruentus & + & + & $\mathrm{R}+\mathrm{B}$ \\
\hline Sulphur-breasted Bush-Shrike & Malaconotus sulfureopectus & + & - & $\mathrm{R}+\mathrm{B}$ \\
\hline Grey-headed Bush-Shrike & Malaconotus blanchoti & + & + & $\mathrm{R}+\mathrm{B}$ \\
\hline \multicolumn{5}{|c|}{ Order Passeriformes: 16- Family: Dicruridae } \\
\hline Drongo & Dicrurus adsimilis & - & + & $\mathrm{R}+\mathrm{B}$ \\
\hline \multicolumn{5}{|c|}{ Order Passeriformes: 17- Family: Corvidae } \\
\hline Piapiac & Ptilostomus afer & + & + & $\mathrm{R}+\mathrm{B}$ \\
\hline Cape Rook* & Corvus capensis & + & + & $\mathrm{R}+\mathrm{B}$ \\
\hline Fan-tailed Raven* & Corvus rhipidurus & + & + & $\mathrm{R}+\mathrm{B}$ \\
\hline Pied Crow & Corvus albus & + & + & $\mathrm{R}+\mathrm{B}$ \\
\hline \multicolumn{5}{|c|}{ Order Passeriformes: 18- Family: Oriolidae } \\
\hline Eurasian Golden Oriole & Oriolus oriolus & + & - & $\mathrm{P}$ \\
\hline African Golden Oriole & Oriolus auratus & + & - & $\mathrm{M}$ \\
\hline Yellow-billed Oxpecker & Buphagus africanus & + & - & $\mathrm{R}+\mathrm{B}$ \\
\hline
\end{tabular}


OrderPasseriformes: 19- Family: Sturnidae

\begin{tabular}{|c|c|c|c|c|}
\hline Greater Blue-eared Starling* & Lamprotornis chalybaeus & + & + & $\mathrm{R}+\mathrm{B}$ \\
\hline Lesser Blue-eared Starling & Lamprotornis chloropterus & + & + & $\mathrm{M}$ \\
\hline Bronze-tailed Starling & Lamprotornis chalcurus & + & - & M \\
\hline Long-tailed Glossy Starling & Lamprotornis caudatus & + & + & $\mathrm{R}+\mathrm{B}$ \\
\hline Ruppell,s Long-tailed Starling & Lamprotornis purpuropterus & + & + & $\mathrm{R}+\mathrm{B}$ \\
\hline Purple Starling & Lamprotornis purpureus & + & + & $\mathrm{R}+\mathrm{B}$ \\
\hline Chestnut-billed Starling* & lamprotornis pulcher & + & + & $\mathrm{R}+\mathrm{B}$ \\
\hline \multicolumn{5}{|c|}{ Order Passeriformes: 20- Family: Passeridae } \\
\hline House Sparrow* & domesticus & + & + & $\mathrm{R}+\mathrm{B}$ \\
\hline Kordofan Rufous Sparrow* & Passer cordofanicus & + & + & $\mathrm{R}$ \\
\hline Chestnut Sparrow & Passer eminibey & + & + & $\mathrm{R}+\mathrm{B}$ \\
\hline Sudan Golden Sparrow* & Passer luteus & + & + & $\mathrm{R}+\mathrm{B}$ \\
\hline Grey-headed Sparrow* & Passer griseus & + & + & $\mathrm{R}+\mathrm{B}$ \\
\hline Desert Sparrow & Passer simplex & + & + & $\mathrm{R}+\mathrm{B}$ \\
\hline Bush Petronia & Petronia dentate & + & + & $\mathrm{R}+\mathrm{B}$ \\
\hline Yellow-spotted Petronia & Petronia pyrgita & + & + & $\mathrm{R}$ \\
\hline $\begin{array}{l}\text { Chestnut-crowed Sparrow- } \\
\text { weaver* }\end{array}$ & Plocepasser superciliosus & + & + & $\mathrm{R}+\mathrm{B}$ \\
\hline \multicolumn{5}{|c|}{ Order Passeriformes: 21- Family: Ploceidae } \\
\hline Grey -headed Social-Weaver & Pseudonigrita arnaudi & + & + & $\mathrm{R}+\mathrm{B}$ \\
\hline White-billed Buffalo-Weaver* & Bubalornis albirostris & + & + & $\mathrm{R}+\mathrm{B}$ \\
\hline Speckle-fronted Weaver* & Sporopipes frontalis & - & + & $\mathrm{R}+\mathrm{B}$ \\
\hline Village Weaver* & Ploceus cucullatus & + & + & $\mathrm{R}+\mathrm{B}$ \\
\hline Abyssinian Masked Weaver* & Ploceus intermedius & + & + & $\mathrm{R}+\mathrm{B}$ \\
\hline Vitelline Masked Weaver* & vitellinus & + & + & $\mathrm{R}+\mathrm{B}$ \\
\hline Northern Masked Weaver* & Ploceus taeniopterus & + & + & $\mathrm{R}+\mathrm{B}$ \\
\hline Speke,s Weaver* & Ploceus spekei & + & + & $\mathrm{R}+\mathrm{B}$ \\
\hline Little Weaver* & Ploceus luteolus & + & + & $\mathrm{R}+\mathrm{B}$ \\
\hline Cinnamon weaver & Ploceus badius & + & + & $\mathrm{R}+\mathrm{B}$ \\
\hline Red-billed Quelea & Quelea & + & + & $\mathrm{M}+\mathrm{B}$ \\
\hline Northern Red Bishop* & Euplectes franciscanus & + & + & $\mathrm{R}+\mathrm{B}$ \\
\hline Black-winged Red Bishop & Euplectes hordeaceus & + & + & $\mathrm{R}+\mathrm{B}$ \\
\hline Yellow-crowned Bishop & Euplectes afer & + & - & $\mathrm{R}+\mathrm{B}$ \\
\hline \multicolumn{5}{|c|}{ Order Passeriformes: 22- Family: Estrididae } \\
\hline Green-winged Pytilia* & Pytilia melba & + & + & $\mathrm{R}+\mathrm{B}$ \\
\hline Red-cheeked Cordon-bleu* & Uraeginthus bengalus & + & + & $\mathrm{R}+\mathrm{B}$ \\
\hline Black-faced Fire Finch & Lagonosticta larvata & + & - & $\mathrm{R}+\mathrm{B}$ \\
\hline Red-billed Fire Finch* & Lagonosticta senegala & + & + & $\mathrm{R}+\mathrm{B}$ \\
\hline Cut-throat Finch* & Amadina fasciata & + & + & $\mathrm{R}+\mathrm{B}$ \\
\hline Common Wax Bill & Estrilda & + & + & $\mathrm{R}$ \\
\hline Black-rumped Wax Bill* & Estrilda troglodytes & + & + & $\mathrm{R}+\mathrm{B}$ \\
\hline Crimson-rumped Waxbill & Estrilda rhodopyga & + & - & $\mathrm{R}$ \\
\hline Silver bill* & Euodice cantans & + & + & $\mathrm{R}+\mathrm{B}$ \\
\hline Order Passeriformes:23-Family: & Viduidae & & & \\
\hline Sudan Broad-tailed Whydah* & Vidua orientalis & + & + & $\mathrm{R}+\mathrm{B}$ \\
\hline Pin-tailed Whydah* & Vidua macroura & + & + & $\mathrm{R}+\mathrm{B}$ \\
\hline Purple Indigo bird & Vidua chalybeate & + & + & $\mathrm{R}+\mathrm{B}$ \\
\hline Green Indigo bird & nigeriae & + & + & $\mathrm{R}$ \\
\hline \multicolumn{5}{|c|}{ OrderPasseriformes: 24- Family: Fringillidae } \\
\hline Yellow-fronted Canary & Serinus mozambicus & + & + & $\mathrm{R}+\mathrm{B}$ \\
\hline Streaky-headed Seedeater & Serinus gularis & + & + & $\mathrm{R}$ \\
\hline White-rumped Seedeater & Serinus leucopygius & + & + & $\mathrm{R}+\mathrm{B}$ \\
\hline Order Passeriformes:15-Family: & Emberiziade & & & \\
\hline
\end{tabular}




\section{ISRAA UNIVERSITY JOURNAL OF APPLIED SCIENCE}

Volume 5 :Issue1, October, 2021

ISSN: 2523-0522

\begin{tabular}{|l|l|l|l|l|}
\hline House Bunting & Emberiza striolata & + & + & $\mathrm{R}+\mathrm{B}$ \\
\hline $\begin{array}{l}\text { Cinnamon-breasted Rock } \\
\text { Bunting* }\end{array}$ & Emberiza tahapisi & + & + & $\mathrm{R}+\mathrm{B}$ \\
\hline $\begin{array}{l}\text { African Golden-breasted } \\
\text { Bunting }\end{array}$ & Emberiza flaviventris & + & + & $\mathrm{R}+\mathrm{B}$ \\
\hline Cretzschmar,s Bunting & Emberiza caesia & + & + & $\mathrm{P}+\mathrm{W}$ \\
\hline
\end{tabular}

On basis of IUCN Red List, the conservation status following www.iucnredlist.org (2016) recognized as follows:

1. "265" bird species as Least Concerned (LC). This comprised $97 \%$ of the recorded birds.

2. The following species Circus macrourus, Buteo tachardus oreophilus, Falco concolor,

Falco vespertinus, Erythropygia rufus were recognized as Near Threatened (NT).

3. The following species Gyps africanus, Gyps rueppellii, Neophron monachus bird species as Critically Endangered (CR).

4. Aquila nipalensis orientalis was the only bird species recorded from the area as Endangered (EN).
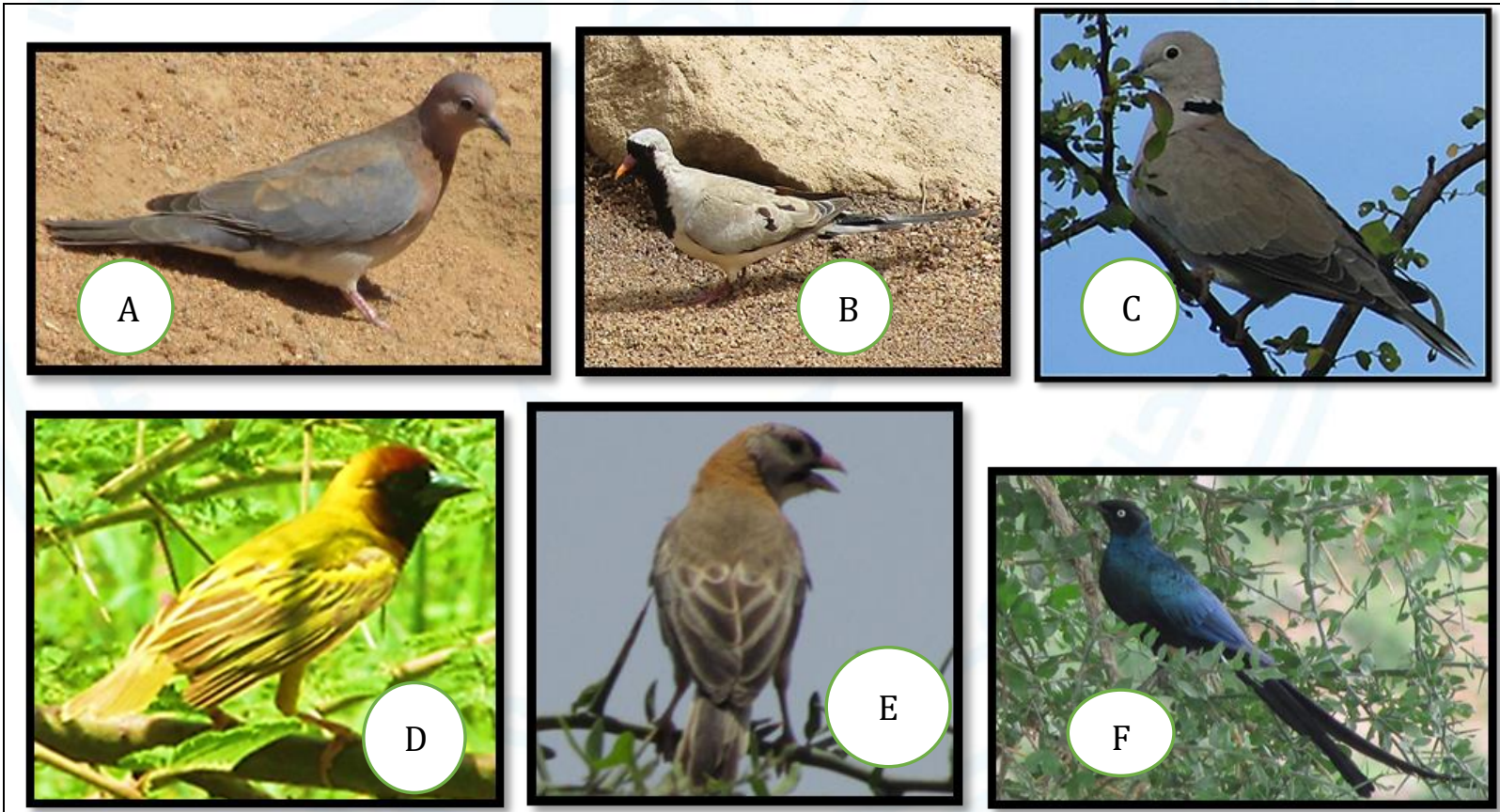

Figure (6): A. Laughing Dove (Streptopelia senegalensis), B. Namaqua Dove (Oena capensis), C. Ring-necked Dove (Streptopelia capicola), D. Northern Masked Weaver (Ploceus taeniopterus), E. Speckle- fronted Weaver (Sporopipes frontails), F. Rueppell's Glossy Starling (Lamprotonis purpuropterus). 

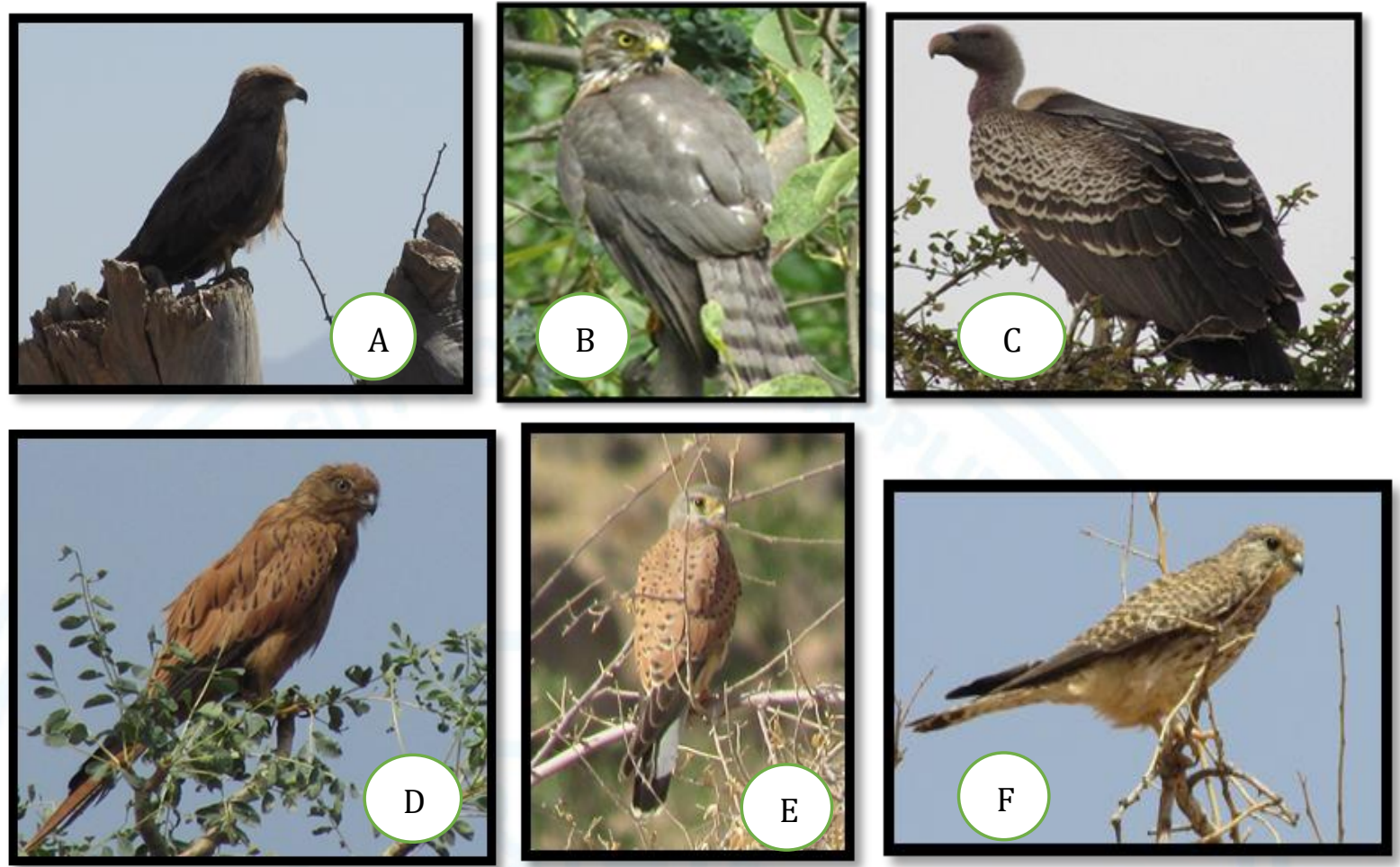

Figure (7): A. Black Kite (Milvus migrans), B. Montagu's Harrier (Circus pygargus), C. Ruppell's Griffon Vulture (Gyps rueppllii), D. Fox Kestrel (Falco alopex), E. Common Kestrel (Falco tinnunculus), F. Lesser Kestrel (Falco naumanni).

Family Passeriformes has highly diverse and abundant with 25 families and 161 species.Psittacidae was represented by two species Poicephalus meyeri and Psittacula krameri (Figure.8).
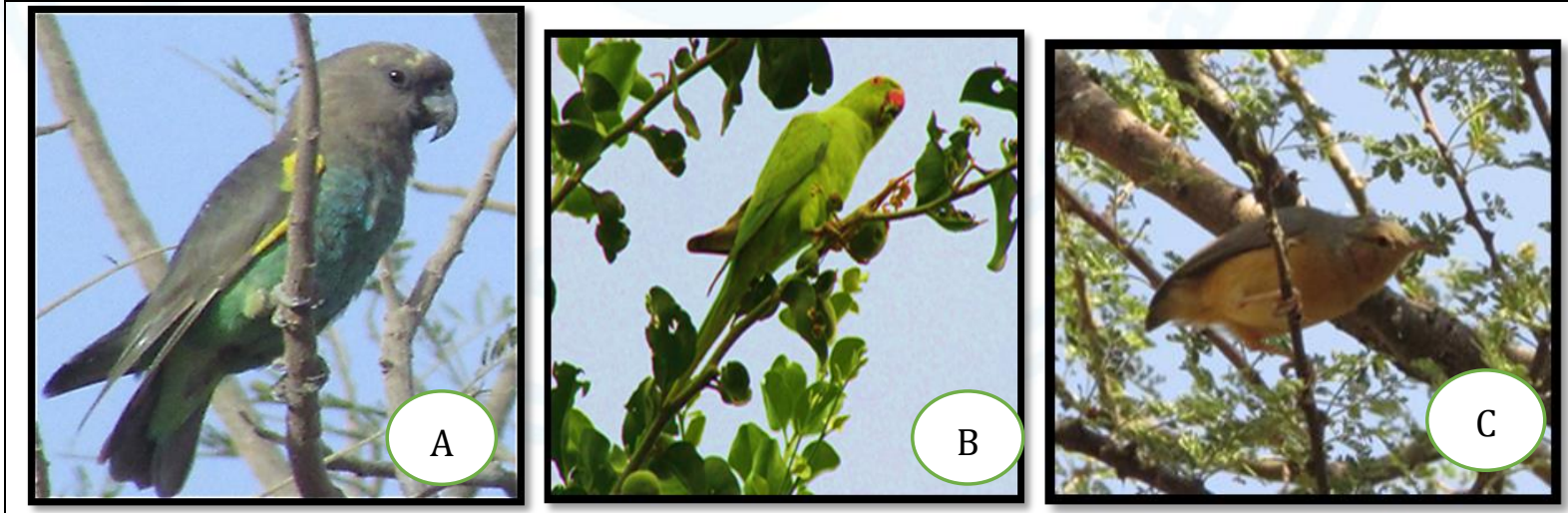


\section{ISRAA UNIVERSITY JOURNAL OF APPLIED SCIENCE}

Volume 5 :Issue1, October, 2021

ISSN: 2523-0522
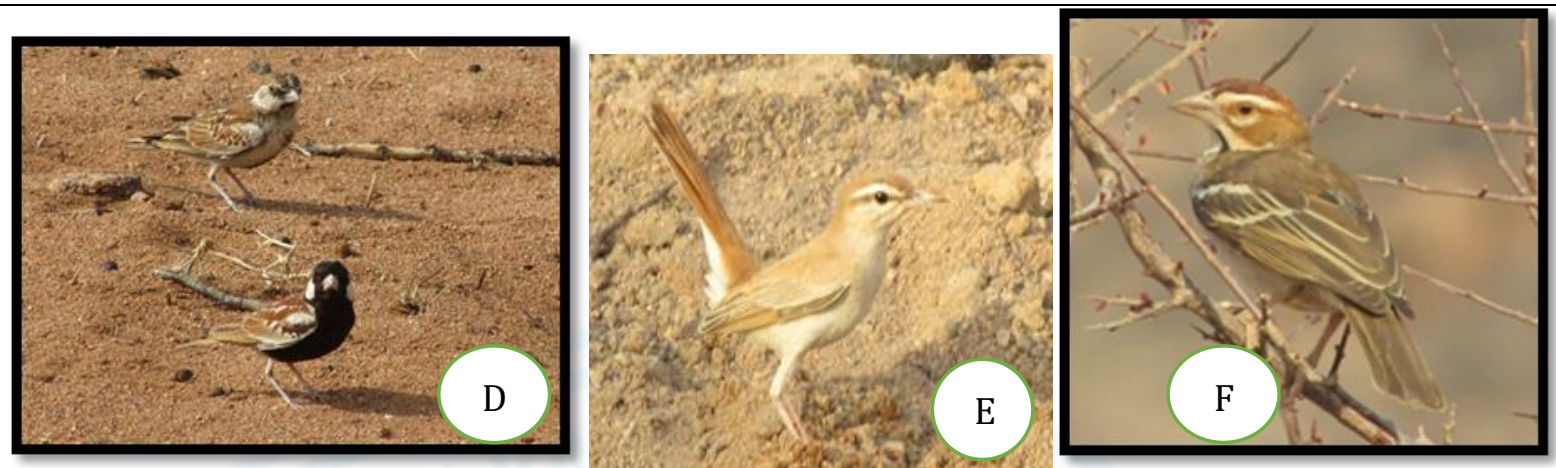

Figure (8): A. Brown Parrot (Poicephalus meyeri), B. Rose-ringed Parakeet (Psittacula krameri), C. Northern Crombec (Sylvietta brachyura), D. Chestnut-headed Sparrow Lark (Eremopterix signata), E. Rufous Bush Chat (Cercotrichas galactotes), F. Chestnut-crowned Sparrow Weaver (Plocepasser superciliosus).

Determinant factors included predators and heat stress (Figure.9,10) and game shooting of Eupodotis ruficrista and Ciconia ciconia.

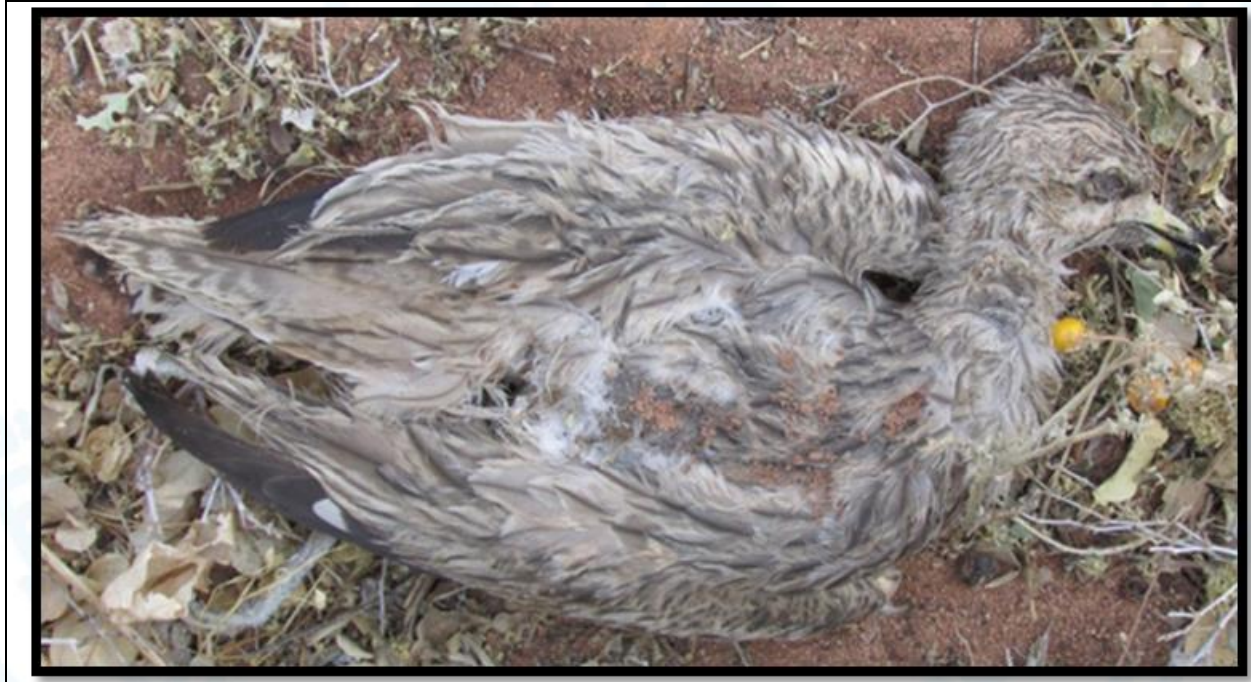

Figure (9): A dead Burhinus senegalensis, December 2015. 


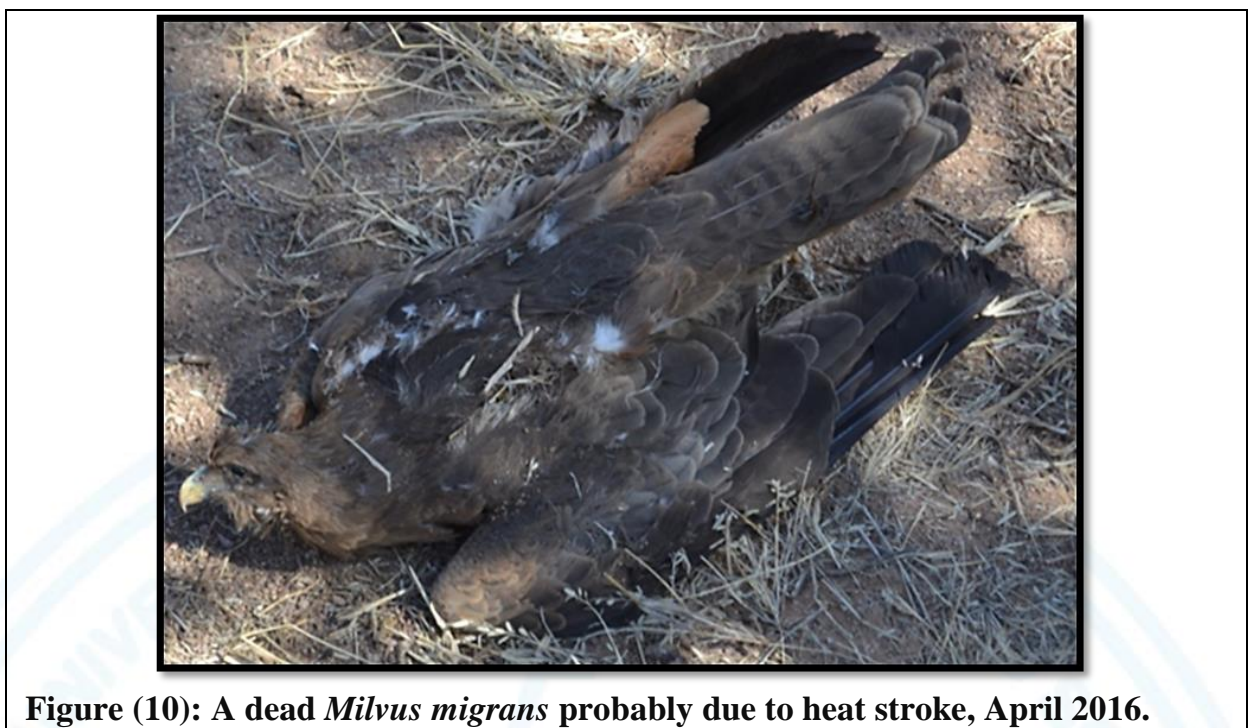

There were marked changes in the bird's fauna in the Jabel El Dayer National Park study area. Between 2014 and 2016 (August 2015 to April 2016). İs a little doubted that these were associated with the climate changes resulting from the onset of rains and subsequent changes in the habitats (Greig-Smith, 1980). The Park is an attractive site for some African (27) and Palearctic (61) migrant species. The African migrants were represented by Clamator glandarius, Coracias naevius, Caprimulgus climacurus and Terpsiphone viridis while the Palearctic were represented by Circus aeruginosus, Falco naumanni, Merops apiaster and Oenanthe pleschanka. This reflects seasonality of the birds in the park. A similar seasonal variation in birds was observed at Dinder National Park, Sudan (Hamed, 1998 and Ramzy, 2010), Shendi Area in the north (Sulieman et al., 2016) and El Ga'ab Depression (Mahmoud et al., 2015). A similar seasonal variation in birds was observed in Palestine by Abd Rabou (2011, 2019,). In fact, the Park in question provided food, resting, nesting, and camouflaging and protection sites for bird fauna. More regular field visits may enrich the bird list of Jabel El Dayer National Park. A birding unit should be established and bird watcher should be encouraged at the Park.

\section{Acknowledgments}

Thanks are due to the late Prof. Dr. Tigani M.H. Allam and to Prof. Dr. Dawi Musa Hamed for supervising this work. I would also like to thank Professor Zuheir Nor El Dauem Mahmood for his help and advises on this work. The thanks reach the hunters and residents of the area who provided some information on bird species during the survey period. 


\section{References}

Abd Rabou, AN. (2019b). Ornithofauna prevailing at Al-Mawasi ecosystem of the Gaza Strip, Palestine. Open J Ecol., 9(9): 360-400.

Abd Rabou NA, Abd Rabou MA. (2019). Notes on the pigeons and doves Family (Cloumbidae) occurring in the Gaza Strip-Palestine. Jordan Journal of Natural History(JJNH), 6(1):30-38.

Abd Rabou, AN. (2011). Notes on some Palestinian bird fauna existing in the zoological gardens of the Gaza Strip. Amer-Eurasian J Agricul Environ Sci., 11(2): 159-172.

Bibby, C, Jones, M and Marsden, S. (1998). Expedition field techniques: Bird surveys. Expedition Advisory Center, Royal Geographical Society (with the Institute of British Geographers), London, pp134.

Cave, F. O. and Macdonald, J. D. (1955). Birds of Sudan. Oliver and Boyd Ltd, Edinburgh \& London.

Dowsett, R.J.; Atkinson, P.W. and Caddick, J.A. (2014). Checklist of the birds of Sudan. Downloaded from www.africanbirdclub.org 7 July.

Evans, M, Amr, Z and Al-Oran, RM. (2005). The status of birds in the proposed Rum Wildlife Reserve, Southern Jordan. Turk J Zool., 29: 17-25.

Greig-Smith, P.W. Ranging behavior of birds in the savannah and riverine forest habitats in Ghana. Ibis, 122: 209-226, 1980.

Guptha MB, Kishore S, Prasad NVS, Rao PVC. Birds of Seshachalam biosphere reserve, South-eastern Ghats of Andhra Pradesh, India. Intl J Biodiv Conserv 7 (12): 435-452, 2015.

Hamed, D. M. (1998). Bird Fauna in Dinder National Park. Sudan Notes and Records, II: 187203.

IFAD and Police of protected wildlife. Jabel ElDayer Management Wildlife Natural Resources. Report (in Arabic), 2008.

IUCN Red List of Threatened Species.ISBN-13:978-098 www.iucnredlist.org 2016.

Mahmoud ZN, Tahir YF, Hamdeen HM. (2015). Birds of El Ga'ab Depression-Sudan. European Academic Research, 3:4408-4415.

Martin, T. E. (1987). Food as a limit on breeding birds: a life history perspective. Annu. Rev. Ecol. Syst. 18: 453-487.

Nickolaus, G. (1987). Distribution atlas of Sudan's Birds with notes on Habitat and Status. Bonner Zoologist monographies, Bonn. No. 25. Zoologists Frostings institute and Museum Alexander Koenig, Germany. 90-322.

Pomeroy, D. Counting birds: AWF Technical Handbook Series 6, African Wildlife Foundation (AWF), Nairobi, Kenya, 48 pp,1992.

Ramzy, A. Y. (2010). Abundance and distribution of Birds in Dinder National Park, Sudan Pakistan J. Wildl., 1(2): 67-72. 
Scott's, J. (2007). East African birds, Singapore by CS Garphics, Second edition. First published in Kenya in 1997 by Kensta, P.O. Box 46309, Nairobi.

Sinclair, L. and Ryan, P. (2010). Birds of Africa South of the Sahara. Struik Nature, Second edition. Princeton University Press, Princeton and Oxford.

Stevenson, T and Fanshawe, J. (2002). Birds of East Africa. CHRISTOPHER HELM. LONDO.

Smith, A. C. Virgl, J. A. Panay, D.I. and Armstrong, A. R. (2005). Effects of a diamond mine on tundra-breeding birds", Arctic, vol. 58, no. 3, Pp.295-304.

Sulieman Y; Pengsakul T, Afifi A, Zakaria M.A. Bird diversity in Shendi area, Sudan. Intl J Res Granthaalayah 4 (6): 55-63,2016.

United Nations Environment Programmer. (2007). Report of the Sudan post-Conflict Environmental Assessment. P.O. Box 30552, Nairobi, KENYA, Tel: +254 (0)20 762 1234, Fax: 254 (0)20 762 3927, E-mail: uneppub@unep.org, Web: http:// www.unep.org, 2007.

Whiteman, A. J. Geology of the Sudan Republic. OxfordUniversity Press, ISBN-13:9780198543732, ISBN-10: 0198543735,1971.

www.google.com/earth/,2016.

www.birdlist.org/sudan.htm.2015.

Wikipedia, Encyclopedia, www.Wikipedia.com. 